\title{
Bacterial Isolates and Semen Analysis of Male Partners of Infertile Couples.
}

\author{
Chika P. Enwuru ${ }^{1}$, Uchenna M. Agbasi ${ }^{2}$, Sarah I. Umeh ${ }^{3,}$ Richard C. Egbuobi ${ }^{4}$ \\ ${ }^{I}$ St Johns Laboratories Limited Owerri, Nigeria. \\ 2 Department of Science Laboratory Technology, Imo State Polytechnic, Umuagwo, Nigeria. \\ ${ }^{3}$ Department of Microbiology, Federal University of Technology, Owerri, Nigeria. \\ ${ }^{4}$ Department of Medical Laboratory Sciences, Imo State University, Owerri, Nigeria.
}

\begin{abstract}
BACKGROUND: Seminal tract infections alter the semen quality and affect the sperm parameters of men with varying degree of effect to male fertility. OBJECTIVES: This study was carried out to investigate the presence of infectious agents other than sexually transmitted infections in semen and their relation to semen parameters. METHODS: Five hundred and eighty-seven semen samples of males investigated for infertility were analyzed, inoculated on sterile culture media, and incubated for $24 \mathrm{hrs}$ at $37^{\circ} \mathrm{C}$. The isolates were identified by standard biochemical tests and their antimicrobial susceptibility determined. RESULTS: Fifty-four percent of the samples had normal sperm concentration, $41.1 \%$ had oligospermia, and $4.9 \%$ had azoospermia. About $64.7 \%$ had normal viscous consistency, $23.0 \%$ were high viscous, and $12.3 \%$ low viscous. Also, $19.4 \%$ had highly motile permatozoa, $51.5 \%$ moderately, $27.6 \%$ poorly and $1.6 \%$ entirely non-motile spermatozoa. About $55.9 \%$ of the samples were infected with different nonspecific pathogens. CONCLUSSION: $55.9 \%$ of the semen samples were infected and this significantly affected the semen parameters. It is necessary to investigate male partners of infertile couples for infections and appropriate treatment initiated.
\end{abstract}

Key words: consistency, infection, morphology, Semen, spermatozoa

\section{Introduction}

Married couples all over the world face varying degrees of infertility [1]. According to the American National Institute of Health, about one third of cases of infertility among married couples is caused by the male factor, another one-third is caused by the female factor. In the remaining one-third, both male and female factors or no apparent cause is detected [2]. In other words, in approximately $40 \%$ of infertile couples, the male factor is either the sole or a contributing cause of infertility. In an attempt to find the possible cause of male factor infertility, semen analysis quickly comes to mind [1]. It is the first and perhaps the most important laboratory investigation of the male partner of an infertile couple [3,4]. Several studies have revealed the role of sperm parameters such as low concentration, poor motility and morphological abnormalities in the infertility situation in males $[5,6]$. These factors are sometimes associated with the presence of non specific seminal tract infections $[5,6,7]$. In our society especially the south eastern part of Nigeria, it is customary for every case of infertile marriage to be blamed on the female partner due to ignorance and a lot of misconceptions about what a fertile man is. Once the man is able to have intercourse, ejaculate semen which he adjudges to be 'thick', then the wife's inability to get pregnant must be a problem with her and not the man. However, with the improvement in the level of education and awareness in our society now, the trend is gradually changing, and many men are now visiting healthcare facilities to verify their reproductive status if they are in doubt. However, there is still the problem of obtaining accurate and reproducible semen analysis and culture results [3]. Standardized technique is not easily applicable in many laboratories performing semen analysis in Nigeria because of unavailability of the approved materials, high cost $[8,9,10]$, or lack of the requisite training to perform correct and acceptable semen analysis [3]. The aim of this study is to determine the presence of bacterial pathogens other than sexually transmitted infections in the semen and its relation to semen parameters in male partners of infertile couples.

\section{Materials and methods}

1.1. The patients: Five hundred and eighty seven semen samples from male partners of couples with problems of fertility referred to St Johns Laboratory Owerri, Imo State in the South-eastern Nigeria from January to December 2010, were used for the study.

1.2. Sample collection: Patients were instructed to wash hands and penis with soap and water, dry hands with sterile disposable towel, then masturbate into a sterile wide mouth leak proof container after 5 days of sexual abstinence and fast from antibiotics. Patients were instructed to ensure that all the ejaculate, especially the first sperm rich portion were included.

1.3. Methods of testing: The semen samples were allowed to liquefy at $37^{\circ} \mathrm{C}$ for 30 minutes before examination. 
1.3.1. Consistency: Each sample was examined macroscopically for consistency according to WHO specification [11] by gently aspirating it into a sterile wide bore (about $1.5 \mathrm{~mm}$ diameter) plastic disposable pipette, allowing the semen to drop by gravity and observing the length of any thread. Any thread longer than $2 \mathrm{~cm}$ is recorded as: a- High viscous; those that appear watery from the moment of ejaculation were recorded as b- low viscous and others as c- Normal viscosity $[12,13]$.

1.3.2. WBC (Leukocytes): A drop of each semen sample was placed on a clean glass slide, covered with cover slip, examined microscopically using 40x objective for the presence of White blood cells and recorded as number of Leukocytes/High Power Field.

1.3.3. Motility: Each undiluted semen sample was filled in an Improved Neubauer counting chamber in replicate and a minimum of 200 cells were counted in each from 5 different areas of the chamber. The percentage motility was graded according to a previous WHO [8] specification as: a- Fast progressive; bSlow progressive; c- Non progressive and d- Non motile. The Progressive Motility Rate was calculated as $\operatorname{PMR}=a+b$, that is fast progressive plus slow progressive and graded as: Highly motile $\{P M R \geq 70 \%\}$, Moderately motile $\{\mathrm{PMR}=40-69 \%\}$, Poorly motile $\{\mathrm{PMR}<40 \%\}$ and Non motile $\{\mathrm{PMR}=0 \%\}$.

1.3.4. Sperm Concentration: Each semen sample was diluted in replicate 1: 20 in a solution of sodium bicarbonate -formaldehyde [12] and filled in an Improved Neubauer counting chamber. The 1 millimeter squares at the four corners of the ruled area were counted and the number of spermatozoa per $\mathrm{ml}$ of semen was calculated. Those with very low spermatozoa concentration were diluted 1:5 or 1:2 and the concentration calculated appropriately [11]. Only intact spermatozoa were counted (spermatozoa with head and tail). Motile pin heads, only heads or headless tails were not counted as prescribed by WHO [11]. Before a semen sample is declared azoospermia (zero spermatozoa concentration), a centrifuged pellet is examined.

1.3.5. Morphology: Smear of the semen sample was made on a grease- free slide, fixed while still wet in $95 \%$ ethanol for 5 minutes, allowed to air-dry, washed in a solution of sodium bicarbonate-formaline and stained [12]. The slide was examined microscopically under oil immersion and percentage normal forms calculated after counting at least 200 cells.

1.3.6. Volume: Each semen sample was measured using a calibrated measuring cylinder and the volume recorded in milliliters.

1.3.7. All the procedures above were carried out in replicate and the results were compared for maximum acceptable differences between replicate results in order to achieve low sampling error according to WHO specification (11).

1.3.8. Culture: Each semen sample was inoculated on sterile blood agar, MacConkey agar and Chocolate agar plates respectively and incubated at $37^{\circ} \mathrm{C}$ for 24 hours. Each plate was examined for evidence of growth and the isolates identified by standard biochemical tests [12].

1.3.9. Antimicrobial susceptibility testing: In-vitro antimicrobial susceptibility testing was performed on each of the isolates by the disk diffusion technique.

1.3.10. Statistical Analysis: Data obtained were analyzed using Chi-square $(\chi 2)$.

\section{Results}

A total of 587 semen samples were analyzed from patients of median age 35.8 years, including some above 60 years of age who are still battling with fertility issues \{TABLE 1$\}$.

1.4. Volume: Thirty seven patients $\{6.3 \%\}$ produced low volume semen sample of $<1.5 \mathrm{ml}, 45$ patients $\{7.7 \%\}$ produced high volume $\{>6.0 \mathrm{ml}\}$ while 505 patients $\{87.0 \%\}$ produced semen of volume $1.5-6.0$ $\mathrm{ml}$ which were within the acceptable volume range.

1.5. Consistency: Three hundred and eighty semen samples $\{64.7 \%\}$ were of normal viscosity, $135\{23.0 \%\}$ were high viscous and $72\{12.3 \%\}$ were low viscous $\{$ watery $\}$. Fig. 1 shows how consistency relates with the sperm concentration.

1.6. Leukocytes: Two hundred and eighty one semen samples $\{47.9 \%\}$ had $\geq 10$ Leukocytes per High Power Field while $306\{52.1 \%\}$ had $<10$ Leukocytes per High Power Field.

1.7. Motility: Ninety nine semen samples $\{19.4 \%$ \} had spermatozoa of Progressive Motility Rate $\{P M R\}$ $\geq 70 \%$ which we consider to be highly motile, $263\{51.1 \%\}$ had Progressive Motility Rate of $40-69 \%$, considered to be moderately motile, $141\{27.6 \%\}$ had PMR $<40 \%$ and considered to be poorly motile[11] while 8 samples $\{1.6 \%\}$ had spermatozoa that were entirely non motile \{necrospermia\}. Fig. 2 shows the Progressive Motility Rate in relation to the presence or absence of bacterial infection.

1.8. Sperm concentration: Twenty nine samples $\{4.9 \%\}$ had no spermatozoa at all \{azoospermia\}, 241 samples $\{41.1 \%\}$ had sperm concentration less than 20 million per milliliter \{oligospermia\}, while 317 samples $\{54.0 \%\}$ had the normal concentration of $\geq 20$ million per $\mathrm{ml}$.

1.9. Morphology: A total of 211 samples $\{41.3 \%\}$ had normal morphology of $\geq 30 \%$ as prescribed by WHO [8]. The frequently observed defects include head abnormalities $=65 \%$ amorphous head, large head, thin 
head, round head and pin head $\}$, neck abnormalities $=30 \%$ \{Bent neck, swollen neck etc $\}$ and tail abnormalities $=5 \%$.

1.10. Culture: Out of the 587 samples analyzed, 537 were cultured. Three hundred of this $\{55.9 \%\}$ were found to be infected with various organisms, $184\{61.3 \%\}$ yielded significant growth of Staphylococcus species including 120 coagulase negative Staph and 64 coagulase positive Staph. Other isolates include Escherichia coli $\{46\}$, Klebsiella spp $\{26\}$, Pseudomonas spp $\{11\}$, Enterococcus spp $\{11\}$, Proteus spp $\{8\}$, Streptococcus spp $\{3\}$ and Candida spp $\{11\}$. About $76.9 \%$ of the azoospermic samples were found to be infected; $58.7 \%$ of the oligospermic samples were infected while $52.1 \%$ of those with normal concentration $\{\geq 20$ million $/ \mathrm{ml}\}$ were infected. Furthermore, $68.7 \%$ of the samples with many leukocytes $\{\geq 10$ cells per High Power Field $\}$ were infected while only $41.8 \%$ of those with less leukocytes were infected. Only 80 of the samples with normal morphology $\{39.9 \%\}$ were infected.

1.11. Antimicrobial susceptibility testing: When tested against the commonly used antimicrobial drugs, 193 isolates $\{66.8 \%\}$ were susceptible to Ofloxacin, $147\{50.9 \%\}$ to Ciprofloxacin and $120\{41.5 \%\}$ to cefotaxime. Seventy four isolates $\{25.6 \%\}$ were resistant to the entire antibiotics used \{TABLE 2$\}$. Most of the multidrug resistant isolates were coagulase negative Staph.

Table 1: Age range variation with sperm concentration.

\begin{tabular}{lllll}
\hline Sperm conc. $\{$ million $/ \mathrm{ml}\}$ & 0 & $<20$ & $\geq 20$ & Total \\
\hline Age Range $\{$ Years\} & 8 & 44 & 87 & $139\{23.7 \%\}$ \\
$\leq 30$ & 15 & 124 & 151 & $290\{49.4 \%\}$ \\
$31-40$ & 4 & 58 & 71 & $133\{22.7 \%\}$ \\
$41-50$ & 2 & 13 & 5 & $20\{3.4 \%\}$ \\
$51-60$ & 0 & 2 & 3 & $5\{0.9 \%\}$ \\
$61-70$ & $29\{4.9 \%\}$ & $241\{41.1 \%\}$ & $317\{54.0 \%\}$ & 587 \\
\hline Total & & & & \\
\hline
\end{tabular}

Table 2: Antibiotic susceptibility test result

\begin{tabular}{|c|c|c|c|c|c|c|c|c|c|c|c|c|}
\hline \multirow{2}{*}{\multicolumn{13}{|c|}{ OFL CPX GEN CTX STR TET AMP CXC ERY NAL NIT None }} \\
\hline & & & & & & & & & & & & \\
\hline $\begin{array}{l}\text { Staph spp } \\
\{\mathrm{n}=184\}\end{array}$ & 117 & 86 & 35 & 72 & 13 & 11 & 12 & 30 & 45 & NT & NT & 59 \\
\hline $\begin{array}{l}\text { E. coli } \\
\{\mathrm{n}=46\}\end{array}$ & 30 & 20 & 0 & 20 & 0 & 5 & 5 & NT & NT & 5 & 28 & 3 \\
\hline Kleb spp & & & & & & & & & & & & \\
\hline$\{\mathrm{n}=26\}$ & 22 & 18 & 4 & 10 & 0 & 0 & 0 & NT & NT & 3 & 12 & 3 \\
\hline $\begin{array}{l}\text { Pseudomonas } \\
\{\mathrm{n}=11\} \\
\text { Enterococcus }\end{array}$ & 8 & 8 & 3 & 3 & 0 & 0 & 0 & NT & NT & 5 & 5 & 3 \\
\hline$\{\mathrm{n}=11\}$ & 6 & 6 & 0 & 5 & 0 & 0 & 5 & 0 & 0 & NT & NT & 5 \\
\hline $\begin{array}{l}\text { Proteus spp } \\
\{\mathrm{n}=8\} \\
\text { Strep spp }\end{array}$ & 8 & 8 & 0 & 8 & 0 & 0 & 0 & NT & NT & 8 & 8 & 0 \\
\hline $\begin{array}{l}\text { Strep spp } \\
\{\mathrm{n}=3\}\end{array}$ & 2 & 1 & 0 & 2 & 0 & 0 & 0 & 0 & 0 & NT & NT & 1 \\
\hline Total & 193 & 147 & 42 & 120 & 13 & 16 & 22 & 30 & 45 & 21 & 53 & 74 \\
\hline
\end{tabular}

$\mathrm{OFL}=$ Ofloxacin, $\mathrm{CPX}=$ Ciprofloxacin, GEN= Gentamycin, $\mathrm{CTX}=$ Cefotaxime, $\mathrm{STR}=$ Streptomycin, $\mathrm{TET}=$ Tetracycline, $\mathrm{AMP}=$ Ampicillin, $\mathrm{CXC}=$ Cloxacillin, $\mathrm{ERY}=$ Erythromycin, $\mathrm{NAL}=$ Nalidixic acid, NIT $=$ Nitrofurantoin, NT= Not tested

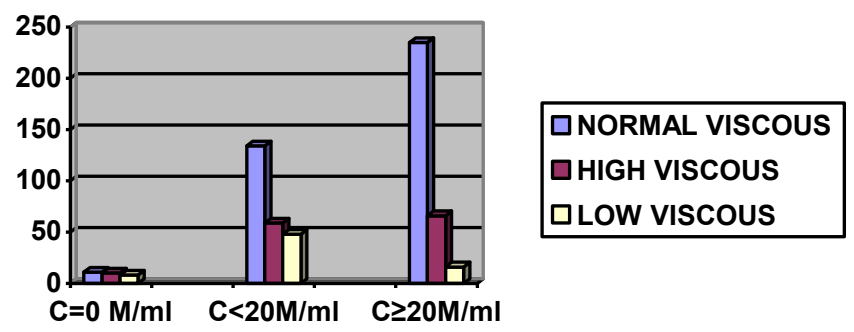

$\mathrm{C}=$ Spermatozoa concentration in millions $/ \mathrm{ml}$ of semen

Figure 1: Number of semen samples with normal viscous, high viscous, or low viscous, consistency for the three grades of concentration (Million per $\mathrm{ml}$ ) of spermatozoa 

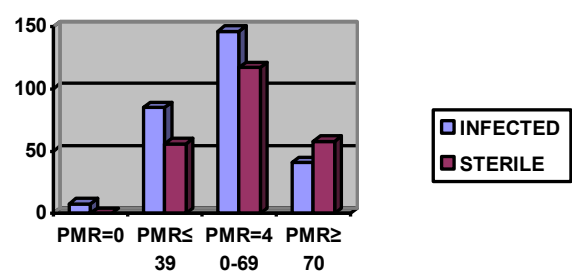

Figure 2: Comparison of infected and sterile semen samples in relation to Progressive Motility Rate (PMR) of the spermatozoa.

\section{Discussion}

The primary aim of semen analysis is to determine the fertility potential of a man as well as to determine the direction to which therapy could be applied to correct observed anomalies where possible. In this study, 37 patients $\{6.0 \%\}$ were unable to produce adequate volume of semen; Forty five patients $\{7.7 \%\}$ produced higher volume than normal. The volume of semen is an important factor in natural fertilization. Low volume results to low total sperm count per ejaculation and too high volume means over dilution of the sperm and low sperm concentration per $\mathrm{ml}$ of semen. This is because the original fluid containing the spermatozoa is small in volume but is diluted by the major fluid produced from the seminal vesicles and the prostate [14]. The WHO normal volume of semen is $2.0-6.0 \mathrm{ml}$.

Twenty three per cent of the entire samples $\{\mathrm{n}=135\}$ were high viscous and some fail to liquefy after 30 minutes. This is a bad sign as the movement of the sperm cells is highly impeded. Also, $12.3 \%\{72\}$ of the samples were low viscous $\{$ watery\}. It had been suggested that altered consistency could be as a result of altered chemical composition of the seminal plasma [5]. Consistency is significantly correlated with sperm concentration $\{\mathrm{p}<0.05\}$. The high viscous and low viscous specimens have lower sperm concentrations than those with normal viscosity $\{$ Fig. 1$\}$.

Furthermore, 281 samples $\{47.9 \%\}$ had $\geq 10$ leukocytes per High Power Field which suggests infection of the seminal tract. Bacteria were isolated from more of those with higher number of leukocytes than from those with fewer leukocytes at the ratio of 1.6: 1 .

This finding is similar to the findings of Mogra et al [5] and Khalili et al [6]. It was also observed that rate of infection is increased in those with decreased Progressive Motility Rate. Similar researches have shown that some bacteria such as E. coli can adhere to the spermatozoa and immobilize them [15]. The Progressive Motility Rate \{Fig. 2$\}$ shows that only $19.4 \%$ of the samples had highly motile spermatozoa $\{\geq 70 \%\}, 51.5 \%$ had moderately motile spermatozoa and $27.6 \%$ had poorly motile spermatozoa while $1.6 \%$ had entirely non motile spermatozoa. This is similar to the findings of Gopalkrishnan [16]. It should be pointed out that motility is one of the most important predictors of male natural fertilization capability [17].

Twenty nine semen samples $\{4.9 \%\}$ were azoospermic \{no spermatozoa at all in the centrifuged pellet of the fluid\}, $241\{41.1 \%\}$ were oligospermic \{less than 20 million spermatozoa per ml $\}$ while 317 samples $\{54.0 \%\}$ had spermatozoa $\geq 20$ million per $\mathrm{ml}$. This higher number of samples with good concentration contrasts with the findings of Mogra [5] who reported only 11.5\% normal concentration, although these two studies vary in space and time. Furthermore, those semen samples with zero sperm concentration \{azoospermia\} could either be as a result of total obstruction of the seminal tract or testicular failure due to endocrine disorder $[18,19]$. Again, it was discovered here that infection was positively associated with low sperm concentration, similar to the findings of Khalili [6] and Noessens [20]. Obstruction of the spermatic duct by scar tissue resulting from infections by both sexually transmitted diseases [18] and non specific seminal tract pathogens have been noted to cause azoospermia.

It was observed in this study that more of the samples with abnormal morphology have more leukocytes and also yielded bacteria growth compared to those with normal forms. This agrees with the conclusion by Berger et al [21] that bacterial infection causes deterioration of spermatogenesis. This also agrees with the findings of Gopalkrishnan [16] and Thomas et al [22]. Sperm morphology has been described as the best predictor of natural fertilization and indicator of quality of spermatogenesis [5].

Furthermore, $55.9 \%$ of the samples cultured $\{300\}$ yielded growth of various bacteria as seen in table 2. This compares to $42.9 \%$ reported by Mogra [5]. The aetiologic agents are also similar but of different frequencies. However, 120 isolates $\{40 \%$ of the whole isolates $\}$ were coagulase negative Staphylococcus which may be contaminants from the microbial flora of the skin and urethral meatus as suggested by Mogra [5]. Some patients find it difficult to produce the semen specimen strictly according to instructions and in the process got the specimen contaminated. However, the microbial flora of the semen of healthy men has not been clearly defined. Organisms such as coagulase negative Staph, Coryneforms, Lactobacilli, Micrococci, Enterococcus 
spp, Strep spp, and E. coli have been reported as members of the normal microbial flora of semen [6,7]. On the other hand, Staphylococcus aureus, Enterococci and some Enterobacteriaceae were isolated from the semen of patients with chronic prostatic syndrome [7] So, whether any isolate from the semen has any clinical significance needs careful evaluation and other factors, such as possibility of contamination during sample collection, considered before a decision is taken.

The antibiogram shows that $66.8 \%$ of the isolates were susceptible to ofloxacin, $50.9 \%$ were susceptible to ciprofloxacin and $41.5 \%$ to cefotaxime. As high as $25.6 \%$ \{74 isolates $\}$ were resistant to all antibiotics used. This is quite alarming, although most of these resistant isolates $\{\mathrm{n}=59\}$ were coagulase negative Staph which we suspect to be commensals from the skin and outer urethra. Nonetheless, the high level of antimicrobial drug resistance observed in this study could be attributed to high level of misuse and over use of antibiotics generally in our society as has variously been reported [23]. All classes of antibiotics are purchased over the counter without doctors' prescription. This results to gross abuse, hence the spread of multidrug resistant strains by selection pressure.

It is important to note that bacteria get access to the seminal tract through the urinary tract. This infected seminal tract and some times the prostate which are inaccessible by many antibiotics in turn act as reservoir of infection to the bladder from time to time. This implies that chronic urinary tract infection is to be expected in some of the patients with significant seminal tract infection.

\section{Conclussion}

In this study, bacterial infections were found in $55.9 \%$ of the semen samples and this was found to have affected significantly the semen parameters and hence the male reproductive potentials. Some non specific pathogens could be present silently in the seminal tract; causing morphological damages to the spermatozoa, reducing the sperm concentration and motility, and causing some biochemical changes in the seminal plasma that affect the overall fertility potential of a man. However, mere presence of bacteria in the seminal fluid does not indicate infection or express ticket to infertility. It is therefore a necessity to investigate the male partner of an infertile couple for the presence of bacteria of proven pathogenic potentials and prompt and appropriate antibacterial therapy instituted following in-vitro antimicrobial susceptibility testing. We recommend that further detailed research be carried out on the causes of the observed significant number of patients with oligospermia and azoospermia respectively so as to curb increasing cases of male infertility.

\section{References}

[1]. Decreases in Male Fertility due to Environmental Factors. Environmental Health Perspectives. Supplement, 101(2): 1993, 65-71

[2]. Male infertility. National Institutes of Health (NIH). Available: http://www.nih.gov/ (accessed 14/03/2013)

[3]. D.R. Franken and O.A. Dada, Does Training Assist Medical Laboratory Scientists with Better Evaluation of Sperm Morphology? African Journal of Reproductive Health 11(1): 2007, 107-112.

[4]. World Health Organization (WHO), Manual for the Standardized Investigation and Diagnosis of the Infertile Couple (Cambridge University Press UK, 1993)

[5]. N.N. Mogra, A.A. Dhruva and L.K. Kothari, Non specific Seminal Tract Infection and Male Infertility: A Bacteriological Study. J. Postgrad. Med; 27: 1981, 99-104.

[6]. M.B. Khalili and M.K. Sharifi-Yazdi, The Effect of Bacterial Infection on the Quality of Human's Spermatozoa. Iranian J. Public Health 30 (3-4): 2001, 119-122.

[7]. I.B. Ivanov, M.D. Kuzmin and V.A. Gritsenko, Micro flora of the Seminal Fluid of Healthy Men and Men Suffering from Chronic Prostatitis Syndrome. International Journal of Andrology 32 (5): 2009, 462-467.

[8]. World Health Organization (WHO) Laboratory Manual for the Examination of Human Semen and Sperm-Cervical Mucus Interaction $4^{\text {th }}$ edn.( UK. Cambridge University Press, 1999)

[9]. D. Mortimer, Practical Laboratory Andrology (Oxford University Press, Oxford, 1994, 71-86).

[10]. T.F. Kruger, R. Menkveld, F.S.H. Stender, C. J. Lombard, J. P. van der Merwe, J. A. van Zyl, and K. Smith, Sperm Morphologic Features as a Prognostic Factor in In-Vitro Fertilization. Fertil. Steril 46: 1986, 1118-1123.

[11]. World Health Organization (WHO) Laboratory Manual for the Examination and processing of Human Semen5th edition. 2010

[12]. M. Cheesbrough, District Laboratory Practice in Tropical Countries Part 2. (Cambridge University Press UK, 2000).

[13]. S.F. Shaban, Male infertility overview. Univ North Carolina School of Medicine, Div Urology, 2007, Available: http://www.ivf.com/shaban.html (Accessed: 22/12/2010).

[14]. S.M. Rosenthal, The fertility Source book (USA. Mc Graw-Hill 1998, Page 96).

[15]. M. R. Auroux, L. Jacques and Mathieu, Is the sperm bacterial ratio a determining factor in impairment of sperm motility: an in-vitro study in men with E. coli. Int. J. Androl. 14: 1991, 264-270.

[16]. K. Gopalkreshnan, R. Joseph and A.R. Sheth, Regulatory Factors in Human Semen with Bacterial Infection. Archives of Andrology 32 (3): 1994, 213-218.

[17]. J.D. Paulson, K.I. Polakoski, Isolation of a Spermatozoal Immobilization Factor from E. coli Filtrate. Fert. Steril 28: 1977, 182-5

[18]. K. Talaro and A. Talaro, Foundations of Microbiology, 2 (USA; WCB/Mc Graw-Hill 1996).

[19]. A. Sharma, A.K Singh, S.K Singh, Increasing Prevalence of Male Infertility and Stress Factors: An Overview. International Journal of Clinical Cases and Investigations 3 (2), 17:24, 2011, 17-24

[20]. A. Naessens, W. Foulon, P. Debrucker and P. Devroeu, Recovery of Microorganisms in Semen and Relationship to Semen Evaluation. Fert. Steril 45: 1986, 101-5.

[21]. R. E. Berger, Epididymitis In: K. K. Holmes, P. A. Mardh, P. F.Sparling and P. J. Wiesner (eds). Sexually Transmitted Dieases (McGraw-Hill, New York. 1984, 650- 662) 
[22]. J. Thomas, S.B. Fishel, J.A. Hall, S. Green, T.A. Newton, and S.J. Thornton, Increased Polymorphonuclear Granulocytes in Seminal Plasma in Relation to Sperm Morphology. Human Reproduction 12: 1997, 2418-2421.

[23]. M. Del Grosso, A. Caprioli, P. Chinazari and M.C. Fontana, Detection and Characterization of Vancomycin-resistant Enterococci in Farm Animals and Raw Meat Products in Italy. Microb. Drug Resis 6: 2000, 313-8. 